\title{
BMJ Gout and the risk for incident heart Open failure and systolic dysfunction
}

\author{
Eswar Krishnan
}

To cite: Krishnan E. Gout and the risk for incident heart failure and systolic dysfunction. BMJ Open 2012;2:e000282. doi:10.1136/bmjopen-2011000282

- Prepublication history for this paper is available online. To view these files please visit the journal online (http:// dx.doi.org/10.1136/ bmjopen-2011-000282).

Received 29 July 2011 Accepted 3 January 2012

This final article is available for use under the terms of the Creative Commons Attribution Non-Commercial 2.0 Licence; see http://bmjopen.bmj.com

Department of Medicine, Stanford University School of Medicine, Palo Alto, California, USA

Correspondence to Dr Eswar Krishnan; e.krishnan@stanford.edu

\section{ABSTRACT}

Objective: To test the hypothesis that gouty arthritis (gout) is a risk factor for incidence of heart failure and for echocardiographic measures signifying subclinical heart failure.

Design: Post-hoc, Iongitudinal and cross-sectional analyses of a prospective cohort study where data were collected in 4-year intervals since 1971.

Settings: The population-based Framingham Offspring Study.

Participants: 4989 adults (mean age 36 years, 52\% women) free of clinical heart failure at baseline.

Outcome measures: Incident heart failure, echocardiographic measures of left ventricular systolic dysfunction, dilatation and hypertrophy.

Results: Participants with gout $(n=228)$ had two to three times higher incidence of clinical heart failure and echocardiographic measures of systolic dysfunction compared with those without. In Cox regression analyses, gout was associated with an adjusted HR of $1.74(95 \% \mathrm{Cl} 1.03$ to 2.93$)$ for incident heart failure and RRs of $3.70(95 \% \mathrm{Cl} 1.68$ to 8.16$)$ for abnormally low left ventricular ejection fraction and of $3.60(95 \%$ $\mathrm{Cl} 1.80$ to 7.72 ) for global left ventricle systolic dysfunction. These risk relationships were consistently observed in all clinical subgroups. Overall, participants with gout had greater mortality than those without (adjusted HR 1.58, 95\% Cl 1.40 to 1.78). Mortality was elevated in subgroup of patients with gout and heart failure (adjusted HR $1.50,95 \% \mathrm{Cl} 1.30$ to 1.73 ) compared to those with heart failure but without gout. Conclusion: Gout is associated with increased risk for clinical heart failure, subclinical measures of systolic dysfunction and mortality.

\section{INTRODUCTION}

Heart failure is a major public health problem in the USA; about 5 million US adults suffer from heart failure with an annual incidence of approximately $550000 .{ }^{1}$ Heart failure is associated with a high risk of morbidity, mortality and hospital utilisation. ${ }^{2}$ The major risk factors amenable to intervention are obesity, hyperlipidaemia, hypertension, diabetes, alcohol abuse and smoking. ${ }^{3}$ A common antecedent for heart failure, atherosclerosis, is also an independent risk factor for gouty arthritis (gout). ${ }^{4-8}$ Patients with gout often

\section{ARTICLE SUMMARY}

\section{Article focus}

- Gout is a common inflammatory arthritis that is a risk factor for cardiovascular disease in general.

- I hypothesised that gout is a specific risk factor for heart failure.

Key messages

- Gout is an independent risk factor for incident heart failure.

- Among those with heart failure gout increases the case death.

\section{Limitations}

- This study does not address the pathophysiological pathways that link gout to heart failure.

n The impact of gout treatment on heart failure risk cannot be assessed.

use medications such as xanthine oxidase inhibitors and non-steroidal anti-inflammatory drug that can decrease or increase the risk for heart failure, respectively. ${ }^{9-13}$ I hypothesised that patients with gout will have a greater risk for clinical heart failure than would be expected from their risk profile. Gout affects over 3.5 million Americans annually. ${ }^{9}$ Hyperuricaemia is necessary but not sufficient for development of gout. ${ }^{14} 15$ Gouty arthritis is characterised by periods of intense inflammatory response with lower grade systemic inflammation in the period between acute attacks. ${ }^{16}$ I prospectively analysed the independent relationship between gout, left ventricular systolic function and incident heart failure in participants in the Framingham Offspring Study (FOS) Cohort. In addition, I sought to study the link between gout and all-cause mortality in the entire cohort and among those who developed heart failure. Being of observational design and consequent inability to account for treatment allocation bias, the analysis of relationship between gout medications such as allopurinol and the risk of heart failure was not included within the scope of the present study. 


\section{METHODS}

Study cohort and data source and design

The FOS is a longitudinal observational cohort assembled in 1971 and includes 5124 men and women who are the offspring of the Framingham Heart Study Cohort and their spouses. ${ }^{17}$ All participants provided informed consent. This study used de-identified data from the FOS obtained through the National Heart, Lung and Blood Institute Limited Access Program that excluded those who did not provide consent for such data sharing and those with unique characteristics that were deemed to be identifiable $(n=4989)$. These individuals were observed over time by periodic examinations approximately 4 years apart; the latest cycle of data collection being in 2008. Data from the medical review, physical examinations and laboratory testing were used for the present analysis. This study is registered at http://clinicaltrials. gov (NCT00005121).

\section{Outcomes}

Clinical Heart failure and mortality

Incidence of heart failure was assessed by questionnaires and by physician interview at the time of follow-up visits. Clinical heart failure and cause of death data were determined predetermined criteria, included in box $1 .{ }^{19} 20$ Specifically, the simultaneous presence of either two major or one major plus two minor criteria, in the absence of an alternative explanation for the symptoms and signs, was required to make the diagnosis of heart failure. Major criteria included the following: paroxysmal nocturnal dyspnoea, orthopnoea, jugular venous distension, hepatojugular reflux, pulmonary rales, radiographic evidence of cardiomegaly, acute pulmonary oedema, third heart sound, central venous pressure above $16 \mathrm{~cm}$ of water and weight loss $>4.5 \mathrm{~kg}$ during the first 5 days of treatment for suspected heart failure. Minor criteria included the following: bilateral ankle oedema, nocturnal cough, dyspnoea on ordinary exertion, hepatomegaly, pleural effusion and heart rate $>120 \mathrm{bpm}$. Hospital and outpatient records were reviewed by a panel of three physicians for adjudication of the heart failure outcomes. There were no participants with heart failure at baseline.

Mortality data were available through the follow-up cut-off date. These included death certificates and the final hospitalisation record where applicable. Information on clinical heart failure was validated by medical chart review. I used all-cause mortality data for our analyses as there was not sufficient power to analyse by individual causes of death.

\section{Echocardiographic measures of left ventricle}

Echocardiographic evaluation was performed on all the available participants at visit $6(\sim$ year $24 ; n=2337)$. Routine transthoracic cardiac echocardiograms with Doppler colour-flow imaging were performed using a Sonos 1000 Hewlett-Packard machine (Andover, Massachusetts, USA). ${ }^{21}$ M-mode measurements of left ventricle (LV) dimensions were performed by a leading

\section{Box 1 Framingham criteria for congestive heart failure ${ }^{18}$}

Major criteria:

Paroxysomal nocturnal dyspnoea

Jugular venous distention

Pulmonary rales

Increasing heart size on chest $x$-ray film

Acute pulmonary oedema

Third heart sound

Central venous pressure of at least $16 \mathrm{~cm} \mathrm{H}_{2} \mathrm{O}$

Hepatojugular reflux

Weight loss of $\geq 4.5 \mathrm{~kg}$ in response to diuretics

Autopsy evidence of pulmonary oedema

Minor criteria:

Bilateral ankle oedema

Nocturnal dyspnoea

Dyspnoea on ordinary exertion

Hepatomegaly

Pleural effusion

Decrease in vital capacity by one-third from prior maximum recorded value

Heart rate $\geq 120 \mathrm{bpm}$

At least 2 major or 1 major plus 2 minor criteria; minor criteria were included only if they were not attributed to another disease process.

edge to leading edge technique according to the American Society of Echocardiography guidelines. ${ }^{18} 22$ Details of echocardiographic measurements including LV mass, LV end-diastolic internal dimensions, LV wall thickness and fractional shortening have been published. ${ }^{23}$ Only those echocardiograms deemed to be of fair or good quality were included in the present analysis. Echocardiographic metrics were treated as continuous and as dichotomous (no abnormality/any abnormality) measures. A validated formula was used to determine LV mass. ${ }^{22-24} \mathrm{LV}$ wall thickness was calculated by adding together the diastolic thicknesses of the septum and the posterior wall. ${ }^{23} \mathrm{LV}$ systolic dysfunction was defined as a fractional shortening of $<0.29 .{ }^{25} \mathrm{In}$ addition, two-dimensional echocardiography was globally assessed by the FOS physician for abnormal ejection fraction and evidence of mild or greater systolic dysfunction as assessed by visual assessment in multiple views. $^{25}$

\section{Assessment of gout}

Gout was defined as a study physician diagnosis and/or use of allopurinol and other gout medications such as probenecid and colchicine definite gouty arthritis. ${ }^{26}$ This case definition is known to have high degree of reliability, ${ }^{24}$ validated using medical records in two large epidemiological studies. ${ }^{24} 27$

\section{Other risk factors of heart failure}

Information on obesity measures, blood pressure, serum lipids, serum glucose, smoking and use of alcohol, 
aspirin, antihypertensive medication and anti-diabetic medication were collected. Hypertension and diabetes mellitus were defined as per standard criteria and by the utilisation of relevant medication. ${ }^{28}{ }^{29}$ For the purpose of this study, participants with a cardiac murmur at the time of the first study visit were assessed to have valvular heart disease. These data were validated by medical record review.

Participants were evaluated for coronary artery disease at baseline and at subsequent visits by medical history, clinician assessment and electrocardiogram.

\section{Medications}

For all medications, current use and past (without specification of time interval) were assessed at the time of the study visits. Information on current use was used for the present study. For our analyses, details of individuals' antihypertensive and diabetes therapy, such as the specific drug, dosage and duration of treatment, were not available.

\section{Renal function}

Based on history and laboratory examination, study physician and staff determined the presence or absence of renal dysfunction. Serum creatinine or other laboratory measures of renal function was not available for the present analysis.

\section{Statistical analysis}

There were three main components for the statistical analysis plan: these included a cohort analysis of gout as a risk factor for heart failure, a longitudinal mortality analysis that assessed the links between gout, heart failure and mortality and finally a cross-sectional analysis of visit 6 data that compared the echocardiographic metrics of those with and without gout. Wherever applicable, all covariates were used as time varying covariates whereby the values of these measures were updated by visits in the statistical models.

\section{Longitudinal analyses for incident heart failure}

Longitudinal data analyses addressed the question of whether gouty arthritis was a risk factor for heart failure. In these analyses, I used Cox proportional hazards regression models where observation time started at baseline or at the time of incident gout and ended on the earliest of the date of incidence of clinical heart failure. Observations were censored at the last available time point in the case of death or loss to follow-up. The Cox model was chosen for heart failure incidence analyses since preliminary examination of the data confirmed the proportionality assumption. The covariates used were selected based on whether they were known risk factors of heart failure: age, body mass index and total cholesterol/high-density lipoprotein ratio as continuous variables and hypertension, body mass index, renal dysfunction, diabetes, alcohol use and smoking as categorical variables.
Longitudinal analyses for mortality risk

These analyses used Poisson regression models where covariates of interest were presence or absence of gouty arthritis; variables adjusted were as above. Cox models were not fitted as the proportionality assumptions were not consistently met.

\section{Cross-sectional analyses for echocardiographic data}

For analyses of echocardiographic data, I used crosssectional analysis methods as these data were obtained only on visit 6 . Ordinary least squared regression models were used to compute adjusted mean echocardiographic measures such as left ventricular thickness. Logistic regression models were used to adjusted estimate proportion of participants with gout and without gout with evidence of left ventricular systolic dysfunction and low ejection fraction. A Poisson approach was used to calculate RRs of dichotomous echocardiographic measures. ${ }^{30}$ These RR estimates are more conservative (smaller magnitude) than would be expected from OR estimates using logistic regression models. ${ }^{30}$ This study was unsponsored. EK possesses raw data, analysis code and will be the guarantor of the scientific integrity of this work. All analyses were performed using STATA (Release 11).

\section{RESULTS}

\section{Heart failure incidence}

Data were available for 4989 FOS participants (figure 1). Table 1 summarises baseline characteristics of the analysis groups used for longitudinal and cross-sectional analyses. None of the participants had heart failure, renal dysfunction or coronary artery disease at the baseline visit. There were 157 individuals who used allopurinol during the course of follow-up; none of these patients developed heart failure and hence effects of allopurinol on heart failure could not be analysed. Table 2 compares the baseline characteristics of participants with gout and without gout within the cohort.

\section{Overall heart failure incidence}

The total observation time was 135991 person-years. The median follow-up time was 15.9 years (IQR 8.1-24.0). Overall, there were 202 incident cases of heart failure. Of these, 187 were associated with hospitalisation and 15 were diagnosed and treated in the inpatient settings. The overall incidence rate $(95 \% \mathrm{CI})$ was $1.5(95 \% \mathrm{CI}$ 1.29 to 1.70$)$ per 1000 person-years. The rates among men and women were 2.2 (95\% CI 1.89 to 2.62) and 0.81 (95\% CI 0.62 to 1.04 ), respectively.

\section{Incidence among participants with gout}

Among those with gout, the incidence of heart failure was 3.5 (95\% CI 2.30 to 5.32) per 1000 person-years. Among men, there were 19 incident cases and among women, there were three. There were no statistically significant differences in incidence rates between men (3.6, 95\% CI 2.3 to 5.6$)$ and women $(3.0,95 \%$ CI 1.0 to 9.2). Since the number of women with gout and heart 
Framingham Offspring Study $\mathrm{N}=5,124$

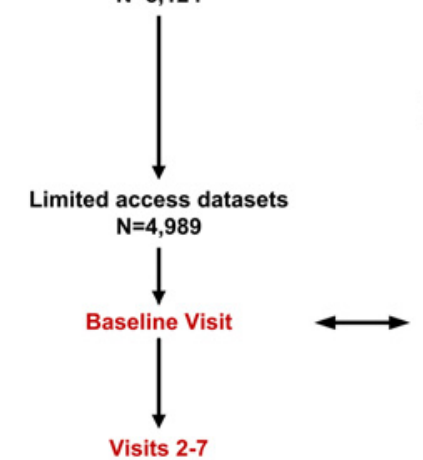

Data from 135 participants not available

Interim onset of comorbidities relevant to heart failure, risk factor measurement, gout attacks, heart failure incidence,

hospitalizations and mortality

Smoking, fasting lipids, glucose and serum urate, blood pressure and anthropometry, information on diagnosis of hypertension, renal disease, diabetes and related medications

Visit 6

Echocardiographic assessment $\mathrm{N}=2,336$

Figure 1 Flow diagram of participants and measurements in the present study. Data from 135 participants were not available either because the characteristics are so unique as to jeopardise de-identification process used in the Limited Access Program or they preferred the data not to be shared with nonFramingham Offspring Study Investigators.

failure were so few, meaningful statistical adjustment in multivariable regressions was not possible and the data were combined for both the genders.
Gout and the risk for incident heart failure

Overall, there were 228 participants with gout. There were relatively few cases of heart failure in the first 10 years of follow-up. Figure 2 shows the Aalen-Nelson cumulative risk curves for heart failure. The curves for subjects with and without gout began to diverge at follow-up year 12 when the mean age of the cohort was 47 years. By year 30 (mean age 66 years), the risk of heart failure was more than twofold higher in the gout group than in the non-gout group.

In unadjusted Cox regression models, gout was associated with an increased risk of heart failure with a HR of 2.8 (95\% CI 1.8 to 4.4$)$. In the multivariable regression models, adjusting for effects of age, hypertension, total cholesterol/high-density lipoprotein ratio, renal dysfunction, diabetes, alcohol use, smoking and body mass index, gout was associated with an increased risk for incident heart failure with a HR $1.75(95 \%$ CI 1.04 to 2.95$)$. Valvular heart disease (ie, presence of heart murmur on clinical examination) was not significantly associated with heart failure incidence, and this variable was not used for multivariable analyses. Gout was a significant predictor of heart failure in the subgroups of patients without hypertension or diabetes and in those with renal impairment.

Table 1 Characteristics of participants included in the analysis

\begin{tabular}{|c|c|c|}
\hline & \multicolumn{2}{|l|}{ Mean \pm SD/per cent } \\
\hline & $\begin{array}{l}\text { For longitudinal analyses } \\
\text { of incident heart failure; } \\
\text { assessed at the first visit }\end{array}$ & $\begin{array}{l}\text { For cross-sectional analyses of } \\
\text { echocardiographic measures of } \\
\text { systolic dysfunction; assessed at } \\
\text { visit } 6 \text { unless otherwise specified }\end{array}$ \\
\hline Number of participants & 4989 & 2336 \\
\hline \multicolumn{3}{|l|}{ Characteristic } \\
\hline Age in years & $36 \pm 10$ & $57 \pm 10$ \\
\hline Proportion of men (\%) & 46 & 45 \\
\hline Body mass index $\left(\mathrm{kg} / \mathrm{m}^{2}\right)$ & $25 \pm 4.3$ & $27.0 \pm 4.3$ \\
\hline Any current alcohol use (\%) & 85 & 62 \\
\hline Proportion of current smokers & 63 & 14.3 \\
\hline Current diuretic users (\%) & 3.2 & 7.3 \\
\hline Systolic blood pressure (mm Hg) & $120 \pm 15$ & $126 \pm 18$ \\
\hline Diastolic blood pressure (mm Hg) & $78 \pm 10$ & $74 \pm 9$ \\
\hline Fasting glucose (mg/dl) & $94 \pm 22$ & $101 \pm 25$ \\
\hline Total cholesterol (mg/dl) & $195 \pm 39$ & $206 \pm 39$ \\
\hline LDL cholesterol (mg//dl) & $124 \pm 35$ & $130 \pm 36$ \\
\hline HDL cholesterol (mg/dl) & $51 \pm 15$ & $52 \pm 16$ \\
\hline Total cholesterol/HDL cholesterol ratio & $4.2 \pm 1.6$ & $1.7 \pm 0.4$ \\
\hline Triglycerides (mg/dl) & $91 \pm 80$ & $134 \pm 100$ \\
\hline Serum urate at visit 1 (mg/dl) & $5.3 \pm 1.3$ & $5.2 \pm 1.3$ \\
\hline Renal disease/dysfunction (\%)* & 3.8 & 3.08 \\
\hline Diabetes $(\%)^{\star}$ & 1.5 & 7.5 \\
\hline Valvular heart disease $(\%)^{\star}$ & 7.3 & 8.1 \\
\hline Current antihypertensive therapy (\%) & 2.4 & 24.3 \\
\hline Gout anytime during study (\%) & 4.6 & 4.1 \\
\hline
\end{tabular}


Table 2 Comparison of participants with and without gout

\begin{tabular}{|c|c|c|c|}
\hline \multirow[b]{2}{*}{ Characteristic } & \multicolumn{3}{|l|}{ Mean $\pm S D / p e r$ cent } \\
\hline & No gout, $N=4291$ & Gout, $N=228$ & p Value \\
\hline Age in years & $36 \pm 10$ & $40 \pm 9$ & $<0.001$ \\
\hline Proportion of men (\%) & 46 & 83 & $<0.001$ \\
\hline Body mass index $\left(\mathrm{kg} / \mathrm{m}^{2}\right)$ & $25 \pm 4.3$ & $28 \pm 4.1$ & $<0.001$ \\
\hline Current alcohol use (\%) & 85 & 95 & $<0.001$ \\
\hline Proportion of current smokers & 63 & 72 & 0.003 \\
\hline Current diuretic use (\%) & 3.2 & 7 & $<0.001$ \\
\hline Systolic blood pressure $(\mathrm{mm} \mathrm{Hg})$ & $120 \pm 15$ & $133 \pm 17$ & $<0.001$ \\
\hline Diastolic blood pressure (mm Hg) & $78 \pm 10$ & $86 \pm 10$ & $<0.001$ \\
\hline Fasting glucose $(\mathrm{mg} / \mathrm{dl})$ & $94 \pm 22$ & $104 \pm 32$ & $<0.001$ \\
\hline Total cholesterol (mg/dl) & $195 \pm 39$ & $213 \pm 38$ & $<0.001$ \\
\hline LDL cholesterol (mg//dl) & $124 \pm 35$ & $138 \pm 34$ & $<0.001$ \\
\hline HDL cholesterol (mg/dl) & $51 \pm 15$ & $45 \pm 14$ & $<0.001$ \\
\hline Total cholesterol/HDL cholesterol ratio & $4.2 \pm 1.6$ & $5.1 \pm 1.7$ & $<0.001$ \\
\hline Triglycerides $(\mathrm{mg} / \mathrm{dl})$ & $91 \pm 80$ & $140 \pm 98$ & $<0.001$ \\
\hline Serum urate $(\mathrm{mg} / \mathrm{dl})$ & $5.3 \pm 1.3$ & $7.3 \pm 1.4$ & $<0.001$ \\
\hline Renal disease/dysfunction $(\%)^{*}$ & 3.8 & 5.8 & 0.14 \\
\hline Diabetes $(\%)^{*}$ & 1.5 & 3.2 & 0.06 \\
\hline Valvular heart disease $(\%)^{\star}$ & 7.3 & 4.4 & 0.1 \\
\hline Current antihypertensive therapy $(\%)^{*}$ & 2 & 8.8 & $<0.001$ \\
\hline
\end{tabular}

\section{Gout and left ventricular dysfunction}

At visit 6, 2237 participants had not developed heart failure and had echocardiograms of acceptable quality available for our analysis. The baseline characteristics of these individuals indicated better health status compared with those who entered the cohort at baseline but did not obtain echocardiogram due to attrition or

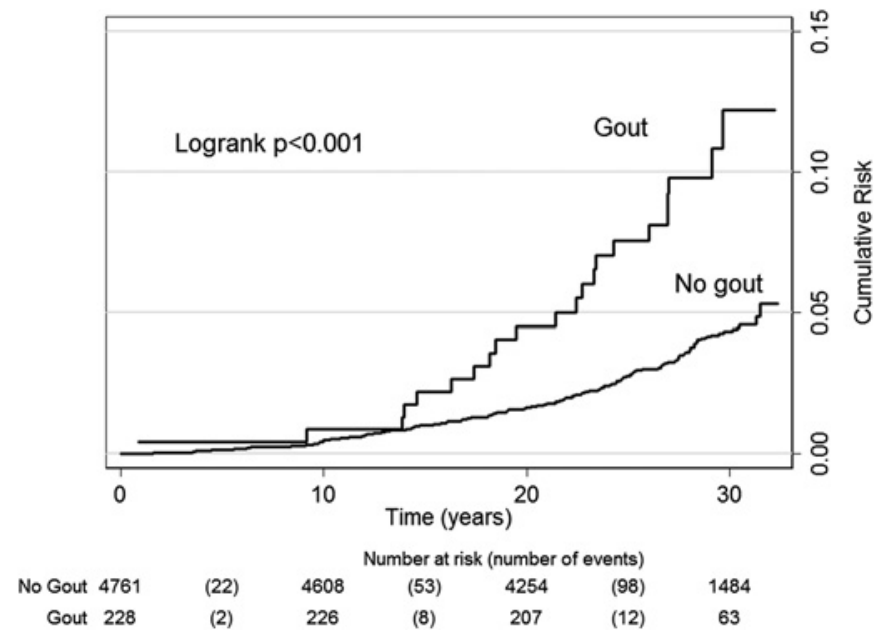

Figure 2 Nelson-Aalen cumulative risk estimates for heart failure among those with and without gout $(n=228$ and 4761 , respectively) in the Framingham Offspring Study. The number of participants at risk for heart failure in each group is provided in two rows. The number of incident cases of heart failure in each time interval is provided within parenthesis. death (table 1). Those with gout had thicker, wider and heavier LVs and had worse indices of LV function after adjustment of covariates (table 3). For the Poisson regression models where the global assessment of $\mathrm{LV}$ function was the dependent variable and gout along with the covariates described in table 4 were the independent variables, patients with gout had a RR of 3.60 (95\% CI 1.80 to 7.18 ) for systolic dysfunction and a RR of 3.70 (95\% CI 1.68 to 8.16) for low ejection fraction.

\section{Mortality analyses of the heart failure group}

Out of the 22 participants in the gout group who developed heart failure, 16 (73\%) died, whereas among the 178 participants with heart failure but no gout, 109 $(61 \%)$ died. Within the gout group, incidence heart failure was associated with substantially higher mortality rate at 95/1000 person-years compared with those without heart failure $8 / 1000$ person-years. Gout was associated with higher mortality rates in unadjusted and adjusted analyses, and this was statistically significant. The magnitude of excess mortality risk associated with gout was not modified by the presence or absence of heart failure table 4 .

\section{DISCUSSION}

Our analysis of data collected on FOS participants indicates that gout is an independent risk factor for subclinical myocardial dysfunction, incident heart failure and mortality after incidence of heart failure. 
Table 3 Echocardiographic characteristics at the Framingham Offspring Study visit $6(\mathrm{~N}=2337)$

\begin{tabular}{|c|c|c|c|c|c|c|}
\hline \multirow{2}{*}{$\begin{array}{l}\text { Echocardiographic } \\
\text { measure }\end{array}$} & \multicolumn{3}{|c|}{$\begin{array}{l}\text { Adjusted for age and body mass } \\
\text { index }\end{array}$} & \multicolumn{3}{|c|}{$\begin{array}{l}\text { Adjusted for age hypertension, } \\
\text { body mass index, renal } \\
\text { dysfunction, diabetes, alcohol } \\
\text { use, smoking and total } \\
\text { cholesterol/HDL cholesterol ratio }\end{array}$} \\
\hline & Gout & No gout & p Value & Gout & No gout & p Value \\
\hline Mean LV thickness $(\mathrm{cm})$ & 2.02 & 1.89 & $<0.0001$ & 1.99 & 1.89 & $<0.0001$ \\
\hline Mean LV fractional shortening (range $0-1$ ) & 0.35 & 0.37 & 0.006 & 0.35 & 0.37 & 0.005 \\
\hline Mean LV diastolic internal dimension $(\mathrm{cm})$ & 5.00 & 4.79 & $<0.0001$ & 4.96 & 4.79 & 0.003 \\
\hline Mean LV mass $(g)$ & 188.51 & 159.29 & $<0.0001$ & 182.47 & 159.58 & $<0.001$ \\
\hline $\begin{array}{l}\text { Proportion of participants with systolic } \\
\text { dysfunction }(\%)^{*}\end{array}$ & 12.7 & 3.5 & $<0.0001$ & 10.0 & 2.3 & 0.002 \\
\hline $\begin{array}{l}\text { Proportion of participants with low ejection } \\
\text { fraction }(\%)^{\star}\end{array}$ & 7.7 & 2.3 & 0.001 & 5.3 & 1.6 & 0.003 \\
\hline
\end{tabular}

This study adds to the growing body of evidence suggesting that gout has major consequences on the cardiovascular system. The cohort studied was large, events were numerous enough for meaningful analyses and the subclinical, clinical and mortality outcomes were well defined. Nevertheless, it is important to keep in mind that data characteristics of FOS could have affected generalisability of our results and conclusions. Our risk estimates may be an underestimate of the true underlying risk for gout since I included allopurinol (a drug with beneficial effect on myocardial systolic function) use as a case definition and since there were no heart failure events among those who took allopurinol. Misclassification of gout diagnosis would have introduced measurement error and reduced the power of this study, that is, type 2 error. There is a concern for residual confounding by factors that were not measured such as the impact of non-steroidal anti-inflammatory drugs often used by patients with gout. Some of the excess risk I observed could be attributed to this class of drugs and not to gout per se. In studies that span 3 decades, competing risks for morbidity and mortality and consequent survivor effects are inevitable. Another important data limitation was that urate levels were measured only in the first two visits and the relative importance of urate and gout could not be assessed. Lastly, information on the duration and severity of gout was not available.

The gout-mortality association I have documented is consistent with prior observations. Studies using data from administrative databases have suggested that among patients with pre-existing heart failure, active gout is associated with $50 \%-100 \%$ excess risk for poor outcomes such as hospitalisation and death. ${ }^{31}$ Tissue hypoxia - a hallmark of heart failure-is a stimulus for

Table 4 Mortality analyses by gout and heart failure status in the Framingham Offspring Study using Poisson regressions $(\mathrm{n}=4989)$

\section{Number of observations} in the model

32267

32267

30774

1493

27209

27209

26073

1136
RR for death $(95 \% \mathrm{CI})$

5.28 (4.89 to 5.69$)$

$1.74(1.57$ to 1.93$)$

1.55 (1.34 to 1.76$)$

1.24 (1.02 to 1.51$)$

3.73 (3.39 to 4.10$)$

1.58 (1.40 to 1.78$)$

1.50 (1.30 to 1.73$)$

1.37 (1.10 to 1.74$)$

Gout vs no gout among those without heart failure

${ }^{*}$ Adjusted for age, body mass index and total cholesterol/high-density lipoprotein ratio as continuous variables and hypertension, body mass index, renal dysfunction, diabetes, alcohol use and smoking as categorical variables. 
the production of urate ${ }^{32}$; among those with heart failure, serum urate concentrations are inversely correlated with maximal oxygen uptake and functional status. ${ }^{33}$ Serum urate levels correlate well with circulating markers of inflammation and with oxidative stress in patients with chronic heart failure. ${ }^{33}{ }^{34}$ Indeed, there is an inverse relationship between serum urate concentrations and peripheral blood flow in patients with chronic heart failure. ${ }^{35}$ Serum urate levels can predict mortality in patients with chronic heart failure. ${ }^{36}{ }^{37} \mathrm{In}$ our study, urate was measured only in first two visits.

The pathophysiological pathways that link gout and myocardial dysfunction are unclear. The two major categories of heart failure are those caused by hypertension and those caused by atherosclerotic coronary artery disease. This study cannot assess the relative contributions of such pathways as the risk factors that cause atherosclerotic heart disease are collinear with those for heart failure. Furthermore, gout is known to be associated with both of these intermediate steps to heart failure. ${ }^{38}$ Hyperuricaemia has been linked to incident heart failure. ${ }^{39}$ Increased serum acid levels may contribute to the echocardiographic abnormalities associated with heart failure through effects on endothelial function and inflammation. In a small study in patients with chronic heart failure $(\mathrm{n}=55)$, the concentration of serum uric acid was an independent predictor of the inflammation markers intracellular adhesion molecule 1, tumour necrosis factor, soluble tumour necrosis factor receptors and interleukin $6 .{ }^{34}$ The National Health and Nutrition Survey conducted in former West Germany also showed an association between serum uric acid concentration and $\mathrm{C}$ reactive protein. ${ }^{40}$ Uric acid can inhibit nitric oxide production by vascular endothelial cells and their proliferation and migration. ${ }^{41}$ Another possibility is that the link might be mediated through hypertension. In an analysis of Framingham Study participants who did not have hypertension, myocardial infarction, heart failure, renal failure or gout at baseline, serum uric acid levels were an independent predictor of hypertension and progression to a higher blood pressure stage. ${ }^{42}$ Finally, the renin-angiotensin system has been proposed to cause left ventricular hypertrophy and cardiac fibrosis through mechanisms including blood pressure increase, direct action of angiotensin II on cardiac myocytes and effects of aldosterone. ${ }^{43}$ Data on these biological factors are not available for the present study but they merit a separate follow-up study. Furthermore, our study cannot assess the specific pathways that link gout and heart failure, such as hypertension, atherosclerotic cardiovascular diseases, drugs, such as anti-inflammatories, and renal dysfunction. I was also unable to tease out the role of serum urate in gout-heart failure link as it was measured only in the first two cycles.

Heart failure is a major health problem in terms of morbidity, mortality and costs. This study provides yet another potentially modifiable risk factor for heart failure. Future studies will need to examine the relationship between gout severity and heart failure. There have been numerous studies that have reported a favourable effect of the gout medication allopurinol (and its metabolite oxypurinol) on endothelial and myocardial function among those with hyperuricaemia. ${ }^{11}$ These molecules have been associated with improved endothelial function in patients with hypercholesterolaemia, ${ }^{44}$ type 2 diabetes with mild hypertension $^{45}$ or chronic heart failure. ${ }^{12}{ }^{46}$ Some studies have shown an improvement in both LV hypertrophy and endothelial function due to treatment with allopurinol. ${ }^{47}{ }^{48}$ Other studies have reported improvements in clinical outcomes of heart failure among patients with hyperuricaemia upon allopurinol treatment. ${ }^{11} 49 \quad 50$ Interventional studies might be able to assess whether allopurinol use can reduce the incidence of heart failure and subsequent poor outcomes.

Acknowledgements The Framingham Offspring Study is conducted and supported by the National Heart, Lung and Blood Institute (NHLBI) in collaboration with the Framingham Study investigators. This manuscript was prepared using a limited access data set obtained from the NHLBI and does not necessarily reflect the opinions or views of the Framingham Study investigators or the NHLBI. This study was not supported by any industrial funding. EK obtained the data sets, analysed the data and wrote the manuscript. Due to the terms and conditions of data user agreement, I am not able to share data.

Funding This research received no specific grant from any funding agency in the public, commercial or not-for-profit sectors.

Competing interests EK has received honoraria, research grants, ad-board fees or consulting fees from the following entities: Ardea Biosciences, UCB, Inc., Centocor OrthoBiotech, URL Pharma, Metabolex, Takeda

Pharmaceuticals and Savient Pharmaceuticals. In the past 5 years, he has held common stocks of Savient Pharmaceuticals. This manuscript does not discuss any proprietary products manufactured by these companies.

Contributors This study was unsponsored. EK possesses raw data, analysis code and will be the guarantor of the scientific integrity of this work.

Provenance and peer review Not commissioned; externally peer reviewed.

Data sharing statement I am unable to share data due to data sharing agreements in place with the National Heart, Lung and Blood Institute.

\section{REFERENCES}

1. Thom T, Haase N, Rosamond W, et al. Heart disease and stroke statistics-2006 update: a report from the American Heart Association Statistics Committee and Stroke Statistics Subcommittee. Circulation 2006;113:e85-151.

2. Gwadry-Sridhar FH, Flintoft V, Lee DS, et al. A systematic review and meta-analysis of studies comparing readmission rates and mortality rates in patients with heart failure. Arch Intern Med 2004;164:2315-20.

3. Folsom AR, Yamagishi K, Hozawa A, et al. Absolute and attributable risks of heart failure incidence in relation to optimal risk factors. Circ Heart Fail 2009;2:11-17.

4. Krishnan E, Pandya BJ, Chung L, et al. Hyperuricemia and the risk for subclinical coronary atherosclerosis - data from a prospective observational cohort study. Arthritis Res Ther 2011;13:R66.

5. Krishnan E, Baker JF, Furst DE, et al. Gout and the risk of acute myocardial infarction. Arthritis Rheum 2006;54:2688-96.

6. Baker JF, Schumacher HR, Krishnan E. Serum uric acid level and risk for peripheral arterial disease: analysis of data from the multiple risk factor intervention trial. Angiology 2007;58:450-7.

7. Choi HK, Curhan G. Independent impact of gout on mortality and risk for coronary heart disease. Circulation 2007;116:894-900.

8. Krishnan E, Svendsen K, Neaton JD, et al. Long-term cardiovascular mortality among middle-aged men with gout. Arch Intern Med 2008;168:1104-10. 
9. Krishnan E, Lienesch D, Kwoh CK. Gout in ambulatory care settings in the United States. J Rheumatol 2008;35:498-501.

10. Anon. Oxipurinol: alloxanthine, Oxyprim, oxypurinol. Drugs $R D$ 2004;5:171-5.

11. Hare JM, Mangal B, Brown J, et al. Impact of oxypurinol in patients with symptomatic heart failure. Results of the OPT-CHF study. J Am Coll Cardiol 2008;51:2301-9.

12. Farquharson CA, Butler R, Hill A, et al. Allopurinol improves endothelial dysfunction in chronic heart failure. Circulation 2002;106:221-6.

13. Page J, Henry D. Consumption of NSAIDs and the development of congestive heart failure in elderly patients: an underrecognized public health problem. Arch Intern Med 2000;160:777-84.

14. Campion EW, Glynn RJ, DeLabry LO. Asymptomatic hyperuricemia. Risks and consequences in the Normative Aging Study. Am J Med 1987;82:421-6.

15. Vitart V, Rudan I, Hayward C, et al. SLC2A9 is a newly identified urate transporter influencing serum urate concentration, urate excretion and gout. Nat Genet 2008;40:437-42.

16. Pascual E. Persistence of monosodium urate crystals and low-grade inflammation in the synovial fluid of patients with untreated gout. Arthritis Rheum 1991;34:141-5.

17. Dawber TR, Kannel WB, Lyell LP. An approach to longitudinal studies in a community: the Framingham Study. Ann NY Acad Sci 1963;107:539-56.

18. Sahn DJ, DeMaria A, Kisslo J, et al. Recommendations regarding quantitation in M-mode echocardiography: results of a survey of echocardiographic measurements. Circulation 1978;58:1072-83.

19. McKee PA, Castelli WP, McNamara PM, et al. The natural history of congestive heart failure: the Framingham study. $N$ Engl J Med 1971;285:1441-6.

20. Levy D, Larson MG, Vasan RS, et al. The progression from hypertension to congestive heart failure. JAMA 1996;275:1557-62.

21. Devereux RB, Casale PN, Hammond IW, et al. Echocardiographic detection of pressure-overload left ventricular hypertrophy: effect of criteria and patient population. J Clin Hypertens 1987;3:66-78.

22. Devereux RB, Alonso DR, Lutas EM, et al. Echocardiographic assessment of left ventricular hypertrophy: comparison to necropsy findings. Am J Cardiol 1986;57:450-8.

23. Lee DS, Pencina MJ, Benjamin EJ, et al. Association of parental heart failure with risk of heart failure in offspring. $N$ Engl J Med 2006;355:138-47.

24. McAdams MA, Maynard JW, Baer AN, et al. Reliability and sensitivity of the self-report of physician-diagnosed gout in the campaign against cancer and heart disease and the atherosclerosis risk in the community cohorts. J Rheumatol 2011;38:135-41.

25. Vasan RS, Benjamin EJ, Larson MG, et al. Plasma natriuretic peptides for community screening for left ventricular hypertrophy and systolic dysfunction: the Framingham heart study. JAMA 2002;288:1252-9.

26. Abbott RD, Brand FN, Kannel WB, et al. Gout and coronary heart disease: the Framingham Study. J Clin Epidemiol 1988;41:237-42.

27. Choi HK, Atkinson K, Karlson EW, et al. Purine-rich foods, dairy and protein intake, and the risk of gout in men. $N$ Engl $J$ Med 2004;350:1093-103.

28. Chobanian AV, Bakris GL, Black HR, et al. The Seventh report of the Joint National Committee on Prevention, detection, evaluation, and treatment of high blood pressure: the JNC 7 report. JAMA 2003;289:2560-72.
29. American Diabetes Association. Diagnosis and classification of diabetes mellitus. Diabetes Care 2009;32(Suppl 1):S62-7.

30. Zou G. A modified Poisson regression approach to prospective studies with binary data. Am J Epidemiol 2004;159:702-6.

31. Thanassoulis G, Brophy JM, Richard H, et al. Gout, allopurinol use, and heart failure outcomes. Arch Intern Med 2010;170:1358-64.

32. Grum CM, Ketai LH, Myers CL, et al. Purine efflux after cardiac ischemia: relevance to allopurinol cardioprotection. Am J Physiol 1987;252:H368-73.

33. Leyva F, Anker S, Swan JW, et al. Serum uric acid as an index of impaired oxidative metabolism in chronic heart failure. Eur Heart $J$ 1997; 18:858-65.

34. Leyva F, Anker SD, Godsland IF, et al. Uric acid in chronic heart failure: a marker of chronic inflammation. Eur Heart $J$ 1998;19:1814-22.

35. Anker SD, Leyva F, Poole-Wilson PA, et al. Relation between serum uric acid and lower limb blood flow in patients with chronic heart failure. Heart 1997;78:39-43.

36. Bettencourt P, Ferreira A, Dias $P$, et al. Predictors of prognosis in patients with stable mild to moderate heart failure. $J$ Card Fail 2000;6:306-13.

37. Bigger JT, Heller CA, Wenger TL, et al. Risk stratification after acute myocardial infarction. Am J Cardiol 1978;42:202-10.

38. Krishnan E. Inflammation, oxidative stress and lipids: the risk triad for atherosclerosis in gout. Rheumatology (Oxford) 2010;49:1229-38.

39. Krishnan E. Hyperuricemia and incident heart failure. Circ Heart Fail 2009:2:556-62.

40. Frohlich M, Imhof A, Berg G, et al. Association between C-reactive protein and features of the metabolic syndrome: a population-based study. Diabetes Care 2000;23:1835-9.

41. Kang DH, Park SK, Lee IK, et al. Uric acid-induced C-reactive protein expression: implication on cell proliferation and nitric oxide production of human vascular cells. J Am Soc Nephrol 2005;16:3553-62.

42. Sundstrom J, Sullivan L, D'Agostino RB, et al. Relations of serum uric acid to longitudinal blood pressure tracking and hypertension incidence. Hypertension 2005;45:28-33.

43. Cowan BR, Young AA. Left ventricular hypertrophy and reninangiotensin system blockade. Curr Hypertens Rep 2009;11:167-72.

44. Cardillo C, Kilcoyne CM, Cannon RO, et al. Xanthine oxidase inhibition with oxypurinol improves endothelial vasodilator function in hypercholesterolemic but not in hypertensive patients. Hypertension 1997;30:57-63

45. Butler R, Morris AD, Belch JJ, et al. Allopurinol normalizes endothelia dysfunction in type 2 diabetics with mild hypertension. Hypertension 2000;35:746-51.

46. Doehner W, Schoene N, Rauchhaus M, et al. Effects of xanthine oxidase inhibition with allopurinol on endothelial function and periphera blood flow in hyperuricemic patients with chronic heart failure: results from 2 placebo-controlled studies. Circulation 2002;105:2619-24.

47. George J, Carr E, Davies J, et al. High-dose allopurinol improves endothelial function by profoundly reducing vascular oxidative stress and not by lowering uric acid. Circulation 2006;114:2508-16.

48. Xu X, Hu X, Lu Z, et al. Xanthine oxidase inhibition with febuxostat attenuates systolic overload-induced left ventricular hypertrophy and dysfunction in mice. J Card Fail 2008;14:746-53.

49. Struthers AD, Donnan PT, Lindsay $P$, et al. Effect of allopurinol on mortality and hospitalisations in chronic heart failure: a retrospective cohort study. Heart 2002;87:229-34.

50. Wei L, Fahey T, Struthers AD, et al. Association between allopurino and mortality in heart failure patients: a long-term follow-up study. Int J Clin Pract 2009;63:1327-33. 